\title{
STUDYING GAMMA-RAY ATTENUATION PARAMETERS FOR EPOXY/ILMENITE RESTORATION MORTAR
}

\author{
M. A. El-Sarraf ${ }^{* 1}$, R. M. Megahid ${ }^{2}$ and W. A. Kansouh ${ }^{2}$ \\ ${ }^{1}$ Radiological Safety Department, Nuclear and Radiological Regulatory Authority, Cairo, Egypt, e- \\ mail: magdsarraf@gmail.com. \\ ${ }^{2}$ Reactor Physics Department, Nuclear Research Centre, Atomic Energy Authority, Cairo, Egypt.
}

\section{Abstract}

This paper demonstrates on evaluation of mass attenuation coefficient, effective atomic number and electronic density for Epoxy/Ilmenite EP/Il $(\rho=2.51 \mathrm{gm} / \mathrm{cm} 3)$ composite of radiation shielding potential required for radiological safety.

Narrow beam gamma-ray transmission geometry was used for the experimental evaluation of mass attenuation coefficient (cm2・g-1). The source term was the radioactive sources Cs-137 and Co-60; the detection term was the $\mathrm{NaI}(\mathrm{Tl})$ scintillation detector. The obtained linear attenuation coefficients from the experimental results were divided by their associated density values to get $(\mu / \rho)$ for the composite. In addition; WinXcom program, as well as, MCNP5 code were used to calculate those parameters theoretically for reasons of comparison and confirmation. The parameters, effective atomic number Zeff and electronic density Nel were experimentally evaluated and theoretically calculated using the first computational tool.

In general, good agreement has been observed between experimental and theoretical methods within uncertainties.

Keywords: Epoxy/Ilmenite composite, mass attenuation coefficient, effective atomic number, electronic density.

\section{INTRODUCTION}

In recent decades, gamma radioactive sources have a variety of uses in modern technology. Their interaction with matter is fundamentals of physics and their harmful effect requires shielding. Recent research is concerned with the creation of polymeric composite with many difficult-to-attain utility properties, as well as, radiation attenuation capabilities. Epoxy filled with ilmenite formulation had been prepared to be used for various radiation shielding purposes and as restoration mortar for nuclear facilities biological concrete shield structures [1,2]. The study of parameters, such as, mass attenuation coefficient $\left(\mathrm{cm}^{2} \cdot \mathrm{g}^{-1}\right)$, effective atomic number $\mathrm{Z}_{\text {eff }}$ and electronic density $\mathrm{N}_{\mathrm{el}}$ is of prime importance in characterization of shielding materials.

We report in this paper the experimental measurements of linear gamma ray attenuation coefficients using Cs-137 and Co-60 sources with gamma energies (0.662, 1.173 and $1.332 \mathrm{MeV})$. The sources strengths are of $1138.808 \mathrm{MBq}$ and $318.618 \mathrm{MBq}$ at the time of experiment, respectively.

Furthermore, the mass attenuation coefficient $\mu / \rho\left(\mathrm{cm}^{2} \cdot \mathrm{gm}^{-1}\right)$ was evaluated by dividing the experimental linear attenuation coefficient $\mu$ by the related composite density. Also, the two theoretical techniques; first, WinXcom [3] computer program developed for calculating mass 
attenuation coefficients for elements, compounds and mixtures for photon energies from $1 \mathrm{KeV}$ to $100 \mathrm{GeV}$ [4] and, second, MCNP5 code [5] were used to evaluate $\mu / \rho$ theoretically for comparison and confirmation.

Gamma rays are related to $\mathrm{Z}_{\text {eff }}$ and $\rho$ as they undertake scattering and absorption reactions upon traversing mixtures and compounds. The effective atomic number is obtained by dividing the total atomic cross-section by the total electronic cross-section. The parameters $Z_{\mathrm{eff}}$ and $\mathrm{N}_{\mathrm{el}}$ have been experimentally evaluated and theoretically calculated $[6,7]$.

\section{EXPERIMENTAL MEASUREMENT OF GAMMA - RAY ABSORPTION}

The experimental arrangement for measuring the linear attenuation coefficient was as the setup found elsewhere [1].

A well-collimated beam from (Cs-137 and Co-60) radioactive sources was used at the source term. At the detection term; we used a $\mathrm{NaI}(\mathrm{Tl})$ crystal with the dimensions $5 " \times 5$ " of energy resolution $8 \%$ at $662 \mathrm{KeV}$. The detector output pulses were amplified then collected via ORTEC (1024 channel) multichannel analyzer (MCA). Finally, the output was processed on a PC-based Maestro-32 software program. Both Cs-137 and Co-60 possesses three gamma quanta; namely the 0.662, 1.173 and $1.332 \mathrm{MeV}$. To obtain the gamma-ray absorption parameter $(\mu)$ for investigated samples, the bare beam intensity $\left(I_{0}\right)$ and I's related to different thicknesses, were measured for a fixed preset time. As well, these counts were selected below a narrow symmetrical region round the centeroid of each photon peak. In addition, the recording time was sufficient to bring the statistical error below $0.3 \%$.

\section{CALCULATION WORK}

\section{Gamma total mass attenuation coefficients $\boldsymbol{\mu} / \boldsymbol{\rho}$}

In good geometric layout i.e. for thin absorber thicknesses and conservation of parallel beams condition, Lambert-Beer law holds and linear attenuation coefficient is as follows:

$$
\mu=\frac{\ln I / I_{0}}{x}
$$

where: $I_{0}$ and $I$ are beam intensities for no sample and sample of thickness $x(\mathrm{~cm})$.

The linear attenuation coefficient $(\mu)$ depends on density $\rho\left(\mathrm{gm}^{\bullet} \mathrm{cm}^{-3}\right)$ which can be variable; therefore it is a common practice to use the mass attenuation coefficient $\mu / \rho\left(\mathrm{cm}^{2} \cdot \mathrm{gm}^{-1}\right)[9,10]$. In our study the computational work for obtaining mass attenuation coefficient has two theoretical roots. First, by using WinXcom Program [3], and second by processing Version 5 MCNP code [5] runs which linear attenuation coefficient outputs will be later divided by the sample density. The used MCNP5 three dimensional code is for modeling the experimental geometry. Related SDEF cards for both sources was used, as well parameters related to $\mathrm{NaI}(\mathrm{Tl})$ material was introduced for the detection cell. In addition DXTRAN sphere technique was used at the detector term to improve the results accuracy and $5 \times 10^{8}$ photon histories were calculated.

\section{Calculation of $\mathbf{Z}_{\text {eff }}$ and $\mathbf{N}_{\mathrm{el}}$}

Theoretical and experimental values of effective atomic number and effective electronic density have been evaluated. These parameters allow the physical characterization of composite. As a first step $\mathrm{Z}_{\mathrm{eff}}$ will be computed using the WinXcom program, according to mixture and compound rule $[6,7,11]$.

$$
\mu_{\mathrm{m}}=\Sigma_{\mathrm{i}} \mathrm{W}_{\mathrm{i}}\left(\mu_{\mathrm{m}}\right)_{\mathrm{I}}
$$

wherew $w_{i}$ weight fraction of each element in composite, $\left(\mu_{m}\right)_{i}$ the mass attenuation coefficient of each individual element in composite.

The values of mass attenuation coefficients were used to calculate the molecular cross-section $\sigma_{\mathrm{t}, \mathrm{m}}$ as follows: 


$$
\begin{array}{r}
\sigma_{\mathrm{t}, \mathrm{m}}=(\mu / \rho)_{\text {composite }} \mathrm{M} / \mathrm{N}_{\mathrm{A}} \\
\mathrm{M}=\Sigma \mathrm{n}_{\mathrm{i}} \mathrm{A}_{\mathrm{i}}
\end{array}
$$

Where $\mathrm{M}$ is the molecular weight, $\mathrm{n}_{\mathrm{i}}$ and $\mathrm{A}_{\mathrm{i}}$ are the number of formula units and the atomic weight of $i^{\text {th }}$ constituent respectively.

$\mathrm{N}_{\mathrm{A}}$ is Avogadro's number.

The total atomic cross-section $\sigma_{\mathrm{t}, \mathrm{a}}$ could be easily determined from the following relation [11]

$\sigma_{t, a}=\frac{\sigma_{t, m}}{\Sigma_{i} n_{i}}=\frac{1}{N_{A}} \sum_{i} f_{i} A_{i}\left(\frac{\mu}{\rho}\right)_{i}(4)$

where $f_{i}=n_{i} / \Sigma_{j} n_{j}$ is the fractional abundance of $\mathrm{i}^{\text {th }}$ element in composite. Also, the total electronic cross-section $\sigma_{\mathrm{t}, \mathrm{el}}$ could be expressed as follows [6,11]

$\sigma_{t, e l}=\frac{1}{N_{A}} \Sigma_{i} \frac{f_{i} A_{i}}{Z_{i}}\left(\frac{\mu}{\rho}\right)_{i}=\frac{\sigma_{t, a}}{Z_{e f f}}(5)$

$$
Z_{e f f}=\frac{\sigma_{t, a}}{\sigma_{t, e l}}(6)
$$

Also, the effective electron number $\mathrm{N}_{\mathrm{el}}$ could be written as follows: [11].

$N_{e l}=\frac{(\mu / \rho)_{\text {composite }}}{\sigma_{t, e l}}(7)$

As a second step, both $\mathrm{Z}_{\mathrm{eff}}$ and $\mathrm{N}_{\mathrm{el}}$ were deduced experimentally. The present work experimentally evaluated mass attenuation coefficient of composite; as well utilized $(\mu / \rho)$ values for elements obtained fromliterature for such purpose $[6,12,14]$.

\section{RESULTA AND DISCUSSION}

The fabricated composite which consist of a number of samples making the total thicknesses ranging from 4.4 to $21.8 \mathrm{~cm}$ were carefully positioned on the sample holder in order to perform the experimental measurements as in figure (1). Figure (2) displays the transmitted gamma rays (photons/sec) at energies $0.662,1.173$ and $1.332 \mathrm{MeV}$ for the concerned composite samples. The attenuation curves show the gamma ray count rate to decrease exponentially with increase in composite thicknesses.

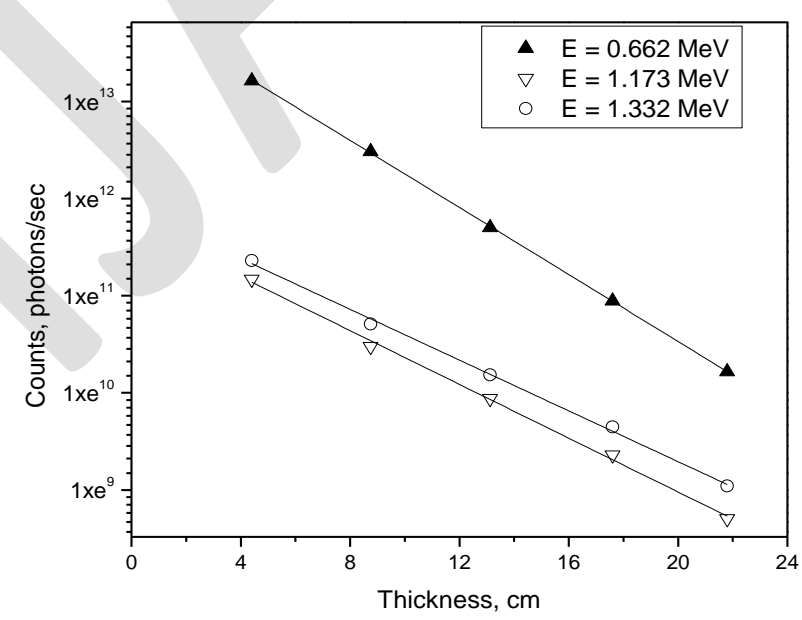

Figure 2. Measured gamma ray counts behind different thicknesses of the composite at 0.662 , 1.173 and $1.332 \mathrm{MeV}$.

Table (2) shows the mass attenuation coefficients values $\mu / \rho\left(\mathrm{cm}^{2} \cdot \mathrm{g}^{-1}\right)$ which are carried out by the experimental evaluation using linear attenuation coefficient $\mu\left(\mathrm{cm}^{-1}\right)$ obtained from figure $(2)$ and the two calculation methods for the three energy lines. It is shown that, a reasonable agreement 
could be observed between the experimentally evaluated and the theoretically computed results. As well, it is clear that the values determined by WinXCom and MCNP5 are very close. This approves and confirms the precision of the MCNP5 model design.

Table 1. Evaluated and calculated values of mass attenuation coefficients for the EpIl composite.

\begin{tabular}{|c|c|c|c|c|}
\hline $\begin{array}{c}\text { Composit } \\
\mathrm{e}\end{array}$ & $\begin{array}{c}\text { Energ } \\
\mathrm{y} \\
(\mathrm{MeV} \\
)\end{array}$ & $\begin{array}{c}\text { Experimental } \\
\text { ly evaluated } \\
\text { mass } \\
\text { attenuation } \\
\text { coefficients, }\end{array}$ & $\begin{array}{l}\text { WinXCo } \\
\mathrm{m} \\
\text { calculated } \\
\text { mass } \\
\text { attenuatio }\end{array}$ & $\begin{array}{c}\text { MCNP5 } \\
\text { calculated } \\
\text { mass } \\
\text { attenuatio } \\
n \\
\text { nooffiniont }\end{array}$ \\
\hline \multirow{3}{*}{ Ep/Il } & 0.662 & 0.06874 & 0.07636 & 0.07388 \\
\hline & 1.173 & 0.05517 & 0.05788 & 0.05554 \\
\hline & 1.332 & 0.05207 & 0.05425 & 0.05220 \\
\hline
\end{tabular}

Basic radiation parameters, namely, mass attenuation coefficient $\mu / \rho\left(\mathrm{cm}^{2} \cdot \mathrm{g}^{-1}\right)$ and mean free path $\lambda$ $\left(\mathrm{cm}^{-1}\right)$ for total and partial interactions (with coherent) is presented for the concerned composite over wide photon energy range $\left(10^{-3}\right.$ to $\left.10^{5}\right) \mathrm{MeV}$ using WinXcom program as shown in figure(3). For total interaction it can be easily seen that there are three energy ranges according to dominance of different partial interaction processes. First comes rapid decrease in $\mu / \rho$ according to prevalence of photoelectric effect, then comes slight decrease according to compton scattering reaction, and last is slight increase for the pair production. As well, the mean free path relation could be divided into three regions; sharp increase followed by slight increase and an end with a light decrease describing the inverse relation of $\mu / \rho$.

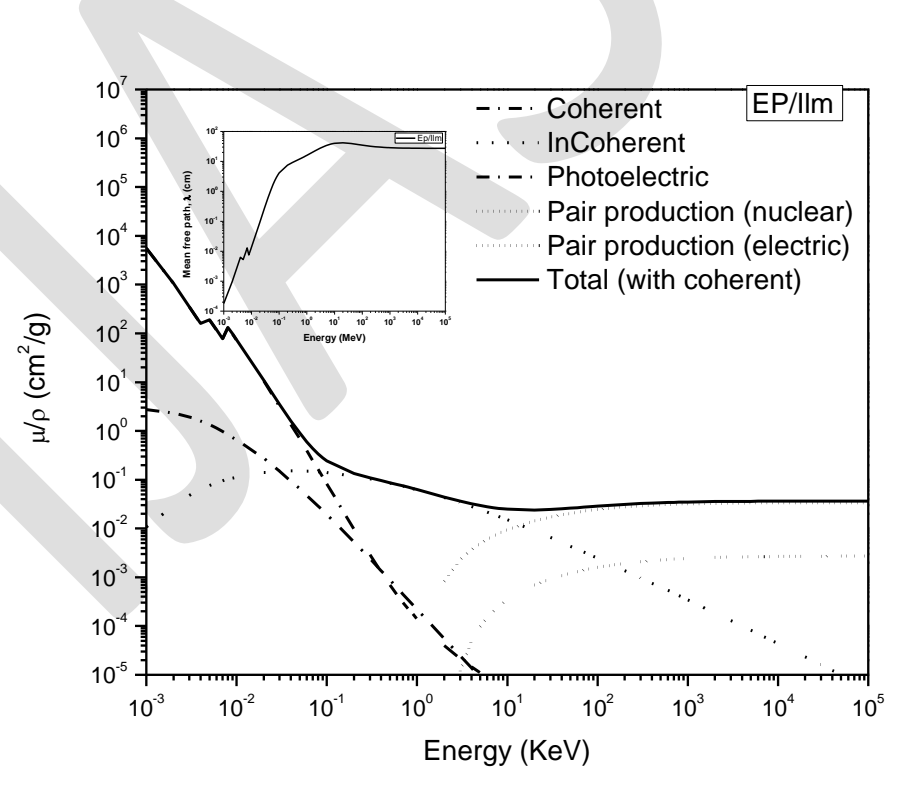

Figure 3. Variation of photon mass attenuation coefficient for EPIl with photon energy for total and partial interaction (with coherent) and variation of mean free path for the same energy range and total interaction.

By using the experimentally determined and the WinXcom theoretically calculated values of $\mu / \rho$, the effective atomic numbers $\left(\mathrm{Z}_{\mathrm{eff}}\right)$ and electron number densities $\left(\mathrm{N}_{\mathrm{el}}\right)$ have been evaluated using equations (6) and (7) respectively and are presented in table (2). It is observed that both 
experimental and theoretical data are very close and the variation with photon energy is very modest for this narrow photon energy region.

Table 2. Measured and calculated values of the effective atomic numbers $\mathrm{Z}_{\mathrm{eff}}$ and electron densities $\mathrm{N}_{\mathrm{el}}$ for the EpIl composite.

\begin{tabular}{|c|c|c|c|c|c|}
\hline \multirow{2}{*}{ Composite } & \multirow{2}{*}{$\begin{array}{c}\text { Energy } \\
(\mathrm{MeV})\end{array}$} & \multicolumn{2}{|c|}{$\mathrm{Z}_{\mathrm{eff}}$} & \multicolumn{2}{c|}{$\begin{array}{c}\mathrm{N}_{\mathrm{el}} \\
\left(\times 10^{23} \text { electron/g }\right)\end{array}$} \\
\cline { 4 - 7 } & & Exp. & Theo. & Exp. & Theo. \\
\hline \multirow{3}{*}{ Ep/Il } & 1.173 & 7.95506 & 8.41337 & 2.78916 & 2.94985 \\
\cline { 3 - 7 } & 0.662 & 7.58164 & 8.44164 & 2.65823 & 2.95976 \\
\cline { 3 - 7 } & 1.332 & 8.06080 & 8.41629 & 2.82623 & 2.95087 \\
\hline
\end{tabular}

In the present study, figures $4(\mathrm{a}-\mathrm{f})$ contain $\mathrm{Z}_{\text {eff }}$ and $\mathrm{N}_{\mathrm{el}}$ graphs in the energy range $1 \mathrm{KeV}-100$ $\mathrm{GeV}$, and it is clear that effective atomic number varies with energy which confirms comment made by Hine 1952 [15].

Both parameters for total and individual interactions is discussed as follows;

a) Total (with coherent) photon interaction,

The behavior of both $\mathrm{Z}_{\mathrm{eff}}$ and $\mathrm{N}_{\mathrm{el}}$ is similar. The investigated composite EpIl is consisted of heavy atomic numbers and at low energy range $(\mathrm{E}<0.01 \mathrm{MeV})$ both parameters increase step by step and give maximum value; where the $\mathrm{Z}^{4}$ dependence for photo electric effect cross-section gives high values for such heavy materials. For intermediate region $(0.05 \mathrm{MeV}<\mathrm{E}<5 \mathrm{MeV})$, where compton scattering is dominant, both parameters decrease with increase in energy. At high energies the parameters goes high again but smaller than low energy range where dominance of pair production has $\mathrm{Z}^{2}$ dependance.

b) Photoelectric absorption,

For this reaction we can observe changes in parameters below (10 MeV) as well. They increase sharply with increase in energy and have no significant variation afterwards.

c,d) Coherent and incoherent scattering,

As shown in figures $(c, d)$ the parameters increase with energy, first in the range $(1-200$ $\mathrm{MeV})$ and second in the range $(1-400 \mathrm{KeV})$ respectively. Afterwards, they become energy independent for the concerned composite.

e,f) Pair production (nuclear and electric field),

From the figures and for both types of reactions we can observe a slight decrease with the 
a)

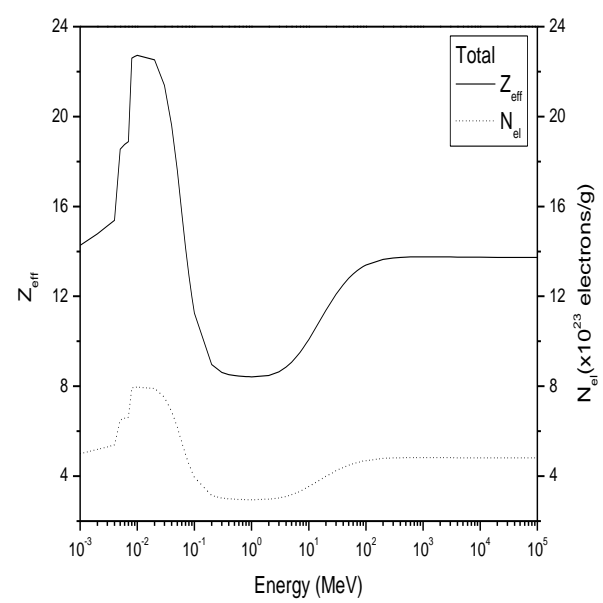

c)

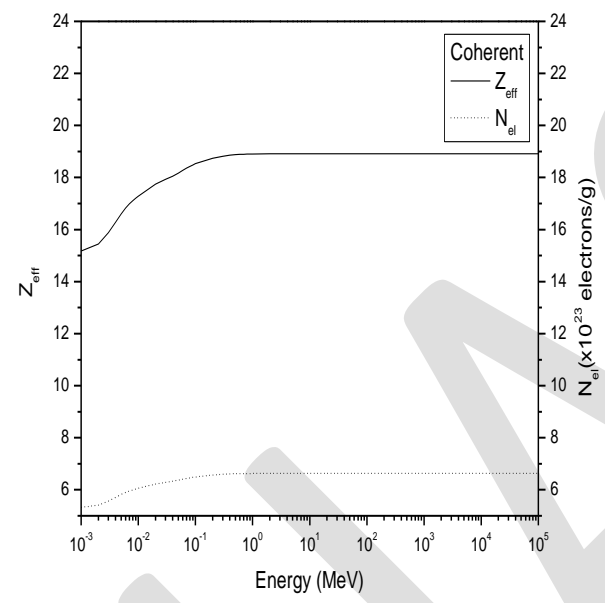

e)

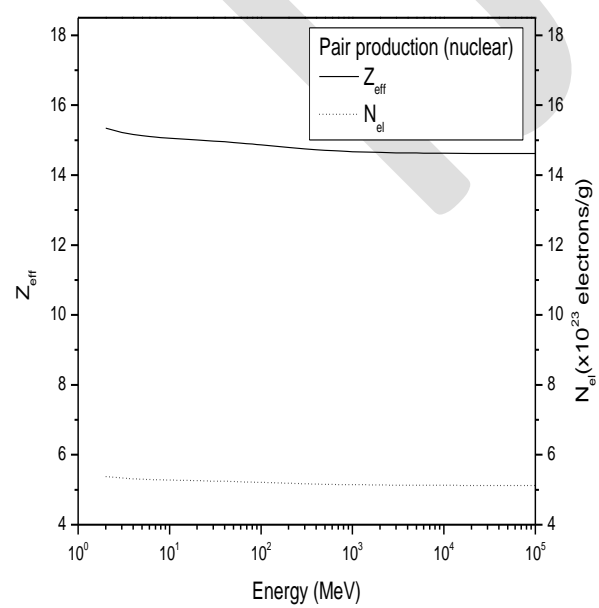

b)

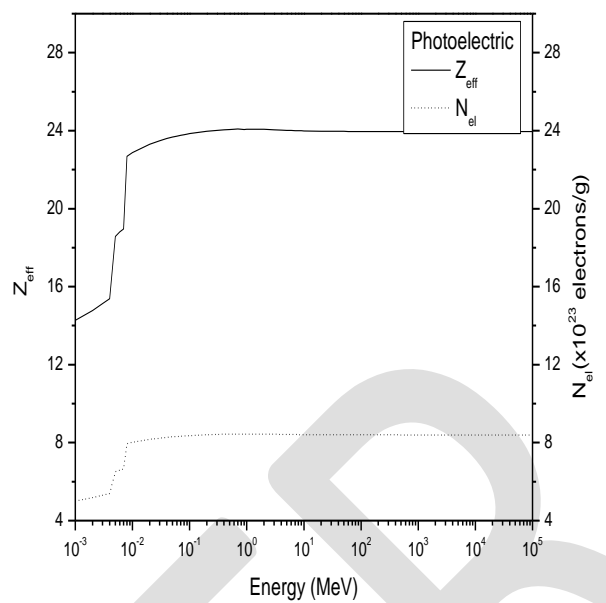

d)

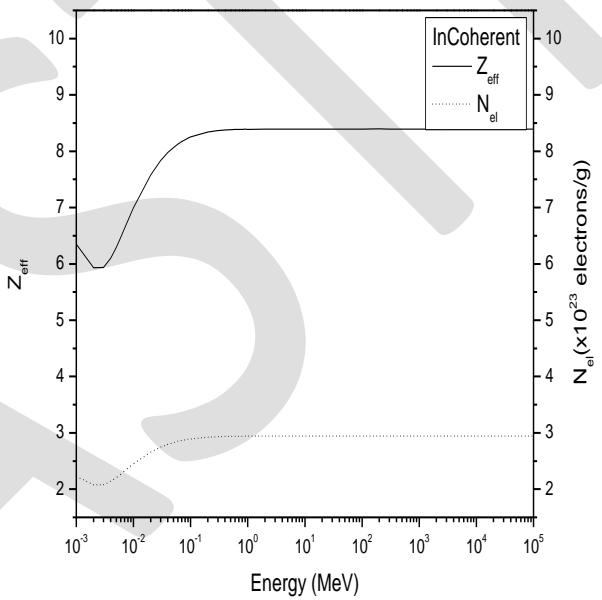

f)

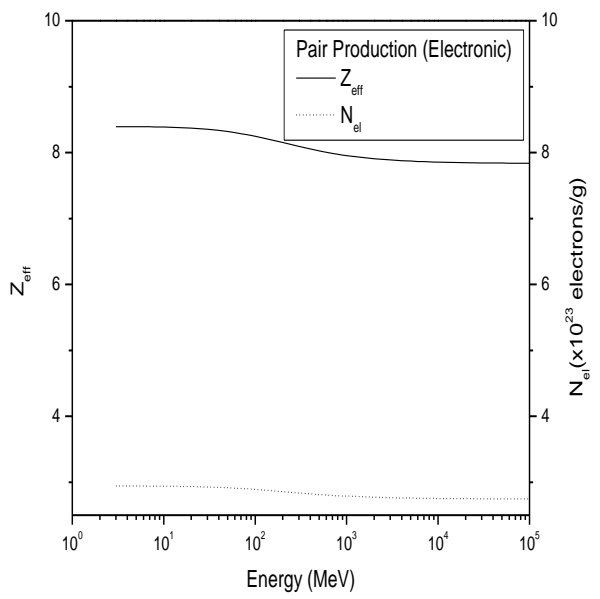

Figure 4. Variation of $\mathrm{Z}_{\mathrm{eff}}$ and $\mathrm{N}_{\mathrm{el}}$ with photon energy for a) total photon interaction-with coherent b) photo electric absorption c) coherent scattering d) incoherent scattering e) pair production in nuclear field f) pair production in electric field. 


\section{CONCLUSION}

The EpIl composite proved to be efficient in terms of the attenuation parameter $\mu / \rho$.The experimental and theoretical calculations using WinXCom and MCNP5 for evaluating $\mu / \rho$ are within reasonable agreement. In addition the theoretically evaluated and experimentally determined values of the closely related parameters $Z_{\text {eff }}$ and $N_{\text {eff }}$ are almost the same and it is clear that both parameters are closely related and are of qualitative energy dependence. And, it is concluded that the investigated composite acquire the potential for radiation shielding.

\section{REFERANCES}

[1] El-Sarraf,M.A., El-Sayed Abdo, A.,Abdul-Wahab, M.A.,Annals of Nuclear Energy,60, 362367 (2013).

[2] El-Sayed Abdo, A., El-Sarraf, M.A., Gaber, F.A.,Annals of Nuclear Energy,30 (2), 175187(2003).

[3] Berger, M.J., Hubbell, J.H., XCOM: Photon Cross Sections Database, Web Version 1.2, available at http://physics.nist.gov/xcom, National Institute of Standards and Technology, Gaithersburg, MD 20899, USA, Originally published as NBSIR 87-3597 ' XCOM: Photon Cross Sections on a Personal Computer', (1987/99).

[4] Gerward, L., Guilbert, N. Jensen, K.B., Levring, H., Radiation Physics and Chemistry,71, pp 653-654 (2004).

[5] X-5 Monte Carlo Team, MCNP - A general Monte Carlo Code for neutron and photon transport, Version 5, Los Alamos National Laboratory(2003).

[6] Limkitjaroenporn, P., Chewpraditkul, W., Kaewkhao, J., Tuscharoen S., Energy Research Journal,2 (1), 29-33 (2011).

[7] Han I., Demir L., Radiation Measurements,44, 289-294 (2009).

[8] El-Sarraf, M.A., El-Sayed Abdo, A., Applied Radiation and Isotopes, 79, 18-24 (2013).

[9] Hubbell, J.H., "Photon Cross Section, Attenuation Coefficients, and Energy Absorption Coefficients From $10 \mathrm{KeV}$ to $100 \mathrm{GeV",} \mathrm{Nat.} \mathrm{Stand.} \mathrm{Ref.} \mathrm{Data.} \mathrm{Ser.,} \mathrm{Nat.} \mathrm{Bur.} \mathrm{Stand.}$ (U.S),29,(1969).

[10] Hubbell, J. H., Phys. Med. Biol.,44, R1-R22 (1999).

[11] Singh, S., Kumar, A., Singh, D., Thind, K. S., Mudahar, G. S., Nuclear Instruments and Methods in Physics Research B,266, 140-146 (2008).

[12] Goswami, B., Chaudhuri, N., Physical Review A,7 (6), 1912-1916 (1973).

[13] Cevik, U.,Baltas, H.,Nuclear Instruments and methods in physics research B, Vol. 256 (2),619-625(2007).

[14] MedhatM.E.,Wang Y., Annals of Nuclear Energy, 80, 365-370 (2015).

[15] Hine, G.J., Phys. Rev.,85, 725(1952). 